\title{
Pattern Recognition Approaches for Classifying IP Flows $^{\star}$
}

\author{
Alice Este ${ }^{1}$, Francesco Gargiulo ${ }^{2}$, Francesco Gringoli ${ }^{1}$, Luca Salgarelli ${ }^{1}$, \\ and Carlo Sansone ${ }^{2}$ \\ ${ }^{1}$ DEA, Università degli Studi di Brescia \\ Via Branze, 38, 25123 Brescia, Italy \\ first. lasteing.unibs.it \\ ${ }^{2}$ Dipartimento di Informatica e Sistemistica, Università degli Studi di Napoli Federico II, \\ Via Claudio, 21 I-80125 Napoli, Italy \\ \{francesco.grg, carlosan\}@unina.it
}

\begin{abstract}
The assignment of an IP flow to a class, according to the application that generated it, is at the basis of any modern network management platform. However, classification techniques such as the ones based on the analysis of transport layer or application layer information are rapidly becoming ineffective. Moreover, in several network scenarios it is quite unrealistic to assume that all the classes an IP flow can belong to are $a$ priori known. In these cases, in fact, some network protocols may be known, but novel protocols can appear so giving rise to unknown classes.

In this paper we propose to face the problem of classifying IP flows by means of different pattern recognition approaches. They have been explicitly devised in order to effectively address the problem of the unknown classes, too. An experimental evaluation of the various proposal on real traffic traces is also provided, by considering different network scenarios.
\end{abstract}

\section{Introduction}

Accurate classification of traffic is at the basis of several techniques that are essential to provide Quality of Service (QoS) guarantees and to face attacks in IP based packet networks. The assignment of a packet flow to the class, from a set of classes that represent all the possible traffic crossing a network edge, is, in fact, requisite to allocate resources according to QoS and security policies. Classical approaches based on port analysis and Deep Packet Inspection are currently implemented on several commercially available products such as firewalls, routers and Application Level Gateways. These devices, that are widely used to engineer Internet traffic, assign flows to classes by thoroughly analyzing the payload of observed packets at the Network and Transport layers. The emergence of new network applications is, however, putting into discussion the reliability of these mechanisms: apart from dynamic port negotiation, several issues arise when existing protocols are used in a way they were not originally conceived for or when protocols

\footnotetext{
* This work has been partially supported by the Ministero dell'Università e della Ricerca in the framework of the PRIN Project "Robust and Efficient traffic Classification in IP nEtworks".
} 
are tunneled one inside another by ad-hoc encapsulation. Indeed the use of obfuscation techniques and encryption mechanisms definitely make payload based approaches completely ineffective: a clear example is given by the latest Peer-to-Peer applications that exploit all these tricks with the specific objective of bypassing the policies at the network boundary [1]. Research efforts have been unceasingly growing in the last few years to counter all these impairments and to restore traffic control capabilities.

Rather than concentrating on deterministic properties such as port numbers or packets payload, recently proposed approaches have been designed to statistically analyze features of network flows, such as combination of packet size, inter-arrival times, flow duration, etc. Several encouraging results have been reported on the application of Pattern Recognition (PR) or Machine Learning (ML) techniques to the problem of traffic classification. Recent works, such as [234], persuaded the scientific community to accept the idea that the behavior of network traffic is so heavily influenced by the underlying application protocols to enable the recognition of traffic classes by observing a very limited set of features using ML or PR techniques. Thanks to these works, several other approaches are now being considered to investigate traffic similarities and to determine by means of both supervised or unsupervised algorithms how to divide flows into classes or clusters depending on the application that has generated them: in particular, the work presented in [5] focused on investigating the performance of several supervised approaches (including Naïve Bayes, Bayesian Networks and Decision Trees) in IP flow classification. In [6] unsupervised approaches based on K-Means, Gaussian Mixture Models and Hidden Markov Model are applied for assigning flows to clusters.

From a PR point of view, the approach considered in [5] corresponds to a standard classification problem where one has to classify a pattern by assigning it to one of a set of known classes. A similar approach has been followed also in [7] where seven classes, each one made up of several protocol applications, have been considered during the training of a Support Vector Machine. In the problem presented in [6], instead, it is assumed that novel classes, which can be considered as unknown, can appear during the testing phase of the system. While the authors in [6] employ some heuristics for labeling a cluster as unknown, an alternative approach can be that of rejecting a pattern that does not fit to any of the known classes [8]. To this concern, it has to be noted that, even if several proposals have been made in the open literature for realizing a classifier system with a reject option, in most cases [9]10] authors consider the need of rejecting samples occurring in the overlap region between two known classes (the so-called confusionbased reject or ambiguity-based reject [11]). In the application context described here, instead, we are interested in the so-called distance-based reject, i.e. in a reject rule that is mainly devised for rejecting distant samples (with respect to the known classes) [11].

Therefore, in this paper we propose three PR approaches based on Support Vector Machines, Gaussian Mixture Models and Decision Trees respectively, that have been explicitly developed for coping with the presence of the unknown classes by means of a distance-based reject. Moreover, since it is also possible to define a network scenario in which users can only use a set of predefined applications, in this paper we also evaluate how the above classifiers perform when all the classes are a priori known. In order to compare the different approaches, we also took into account an aspect that seems to be disregarded in the open literature on IP flow classification. Different scenarios, in 
fact, exist in network management. Each of them corresponds to different costs that can be assigned to the different kinds of errors that can be made by the considered classification system. So, an effective comparison among different approaches can be made only after fixing a specific network scenario for which a cost matrix has been defined. To this aim, we present three different network scenarios, two of which refer to the presence of unknown classes, for IP flow classification and propose a cost matrix for each of them.

The rest of the paper is organized as follows: the three PR approaches proposed for IP flow classification are presented in Section 2, The considered network scenarios are illustrated in Section 3 together with some guidelines for defining the corresponding cost matrices. Then, an experimental comparative evaluation of the three approaches on real traffic traces is reported in Section 4 Finally, some conclusions are drawn.

\section{Three Approaches for Classifying IP Flows}

The basic traffic unit we consider in this paper is the flow, that we define as bi-directional ordered sequence of packets with the same IP addresses and TCP port numbers. The only flows we select have a complete and valid opening TCP three-way-handshake and terminate with a complete TCP shutdown or when a packet with RST flag set is seen.

For each flow we extract a feature vector $\mathbf{x}=\left(x_{1}, x_{2}, \ldots x_{n}\right)$ containing the payload sizes of the first packets in the same order as they are seen by the monitored network node at which we want to perform the classification. We leave off packets without payload because they are mostly used to exchange connection state information, for example to set up the connection or to acknowledge the reception of data, and their features give us less information on the specific application protocol the flow carries. To preserve the information of the direction in which a packet travels, the corresponding feature value is multiplied by -1 if the packet is moving from the server to the client, while it keeps its sign if it is sent by the client.

We choose the above features for the identification of these classes because payload size should only depend on the finite state machine that drives the application layer protocol, especially in the early steps of the exchange.

Finally, with the term class in this paper we refer to the set of flows generated by the same application-layer protocol, such as HTTP, POP3 or SMTP protocols.

In the following, for each considered classification technique, we present both an architecture able to handle the case in which all classes are a priori known and an architecture that can address the problem of the unknown classes, by using a suitably defined distance-based reject option. Note that differently from [6], in our approaches we consider only supervised techniques, since we can use a labeled dataset that can be divided in a training set and a test set.

\subsection{SVM-Based Classifier}

The first architecture we present is based on Support Vector Machines (SVM) [12]. As stated in the introduction, it has been yet fruitfully employed in IP flows classification when all classes are a priori known. For the applications that do not contemplate the presence of unknown protocols we choose to use a multi-class one-against-all SVM 
with a Gaussian kernel function. In this case, the regularization coefficient, the kernel parameter and the size of the feature vectors $n$ have been chosen by maximizing the accuracy of the classification on the training samples.

To solve the problem of unknown protocol identification we have designed an alternative architecture, using the one-class SVM proposed by Schölkopf et al. in [13]. The classifier trains a one-class SVM model for each known protocol class building a surface that contains the training observations with a reliability level determined by the model parameters. The one-class models need as input only the samples of the corresponding training class. We use also the flows of the other known training classes for selecting the values of the parameters that allow to identify correctly the flows of the trained class and to rule out the samples of the other classes.

In order to classify a flow, our classifier finds what surfaces, among the ones calculated during the training phase, the corresponding feature values fall in: if there is only one candidate surface, then the flow is assigned to the corresponding class (i.e., application protocol). If, instead, the flow falls outside all the available surfaces, i.e. it is distant from the all the target classes, the flow is marked as not generated by any of the known applications. In some cases it may happen that the flow falls inside the surfaces of two or more different classes: to assign the flow in these cases, we use a multi-class SVM stage like that we described above.

\subsection{GMM-Based Classifier}

The second classifier we adopt is founded on Gaussian Mixture Models (GMM) [14]. This choice is related to the fact that it is quite simple to adapt the classifier so as to address the problem of unknown classes.

In the training phase this classifier trains a GMM for each protocol in the feature space. The size $n$ of the feature vectors is chosen equal to four, following also the choice suggested in [6]. This model approximates the distribution that generates the training flows of a protocol as a linear combination of $L$ basic Gaussian distributions.

The distribution parameters have been computed from the training set using the Expectation Maximization (EM) algorithm [14], while the number of Gaussians $L$ has been determined for each protocol minimizing a parameter that represents the Minimum Description Length (MDL) [15]. This quantity depends on the number of model parameters and on the value of the maximum likelihood computed on the training data, for a given number of Gaussians.

During the test phase, the classifier selects the class that maximizes the a posteriori probability of the protocol that has likely generated the flow. When the classifier needs to detect unknown protocols we introduce a threshold parameter. The threshold is used during classification to assign a testing flow adopting a distance-based reject option: when all the a posteriori probability values calculated for the flow are lower than the threshold, we label it as unknown. The choice of the threshold value entails a trade-off between the outlier identification and the correct recognition of the samples belonging to the training classes: it is, in fact, set by maximizing the overall classification accuracy, including also the correct recognition of the flows that do not belong to any of the known classes. To this end we introduced a new class, that we name other, representing 
a subset of the unknown classes: it is, in fact, assembled using a little subset of the test set composed of only flows that do not belong to any of the known protocols (we cannot use the training set for this purpose as it lacks this kind of samples). As a consequence, the resulting other class represents samples of unknown protocols and is only used to tune the threshold value during the above described maximization step together with the other classes in the training set, using the same weight for each application protocol class.

\subsection{Decision Tree-based Classifier}

The third classifier model is based on a Decision Tree [14]. This model has been chosen since its classification speed makes it very effective for an on-line implementation [5]. To cope with the presence of the unknown class we also defined a suitably reject option, as it will be illustrated in the following. As in the previous case, the size $n$ of the feature vectors was fixed equal to four.

In case of known classes we used a standard C4.5 model; more precisely, the J48 implementation from the Weka library 1 . When considering the presence of known classes only, we used a post pruning method to reduce the tree size and increase prediction accuracy. The pruning method is based on the MDL principle.

For addressing the presence of the unknown classes, we instead considered an unpruned version of the tree. We then added a reliability value to each leaf. Let us suppose that the DT attributes the $j$-th leaf to the $i$-th class: the reliability value of the $j$-th leaf is therefore calculated, in the training phase, as the ratio between the number of the training set samples of the $i$-th class that fall in the $j$-th leaf and the number of training set samples belonging to the $i$-th class.

In this way, a low reliability is associated to leafs which few samples of the correct class fall in. These leafs are then representative of regions of the feature space that should be quite distant from the ones in which the most part of the samples lies. So, after the training phase, we set a threshold in order to reject samples that fall in leafs whose reliability value is below such a threshold. These samples are considered as belonging to the unknown class since are reasonably distant from the target classes.

As in the GMM case, we used a little subset of the test set for setting the threshold so as to optimize the overall accuracy of the DT, including the recognition on the class other.

\section{The Considered Network Scenarios}

\subsection{Known Classes}

We evaluate the performance of the considered approaches in a first scenario where only a fixed set of known protocols have been allowed within the workstations connected by a Local Area Network (LAN) to the Internet. In this scenario each application protocol is described by a class and the union of the $M$ allowed classes represent all the possible

\footnotetext{
${ }^{1}$ Weka is an open source collection of data-mining tools and is freely available at the website http://www.cs.waikato.ac.nz/ml/weka
} 
Table 1. A possible protocol categorization in a QoS context

\begin{tabular}{|c|c|l|}
\hline Priority class & Service & Applications/Protocols \\
\hline \hline 1 & online gaming & HalfLife, Counter-Strike, Age of Empires, etc. \\
\hline 2 & voip & skype, WengoPhone, Ekiga, Asterisk \\
\hline 3 & streaming & mms (wmp), real, quicktime, shoutcast, etc. \\
\hline 4 & network mgmt & dns, netbios, smb, snmp, ntp \\
\hline 5 & interactive & http, https, ssh, ftp(control) \\
\hline 6 & chat & IRC, msn messenger, yahoo messenger, AIM \\
\hline 7 & mail & smtp, pop3, pop3s, imap, imaps \\
\hline 8 & news & nntp \\
\hline 9 & bulk & ftp(data), sftp (data) \\
\hline 10 & p2p & FastTrack, eDonkey2000, BitTorrent, Gnutella, etc. \\
\hline 11 & other & \\
\hline
\end{tabular}

traffic that can cross the edge router of the LAN. This scenario appears in networks where the workstations are strictly controlled by administrators and users cannot install new software: the set of network software suites installed on these workstations determines the $M$ allowed protocols. Even in this scenario, however, traffic classification could be essential for QoS deployment. Given, in fact, that users can configure applications for retrieving data from remote hosts, there could be no correspondence between Transport Layer ports and underneath application protocols: e.g. a user can configure its mail client to send messages through an external provider by connecting to the mail exchanger on port 587; another user can connect to its home gateway to retrieve web information on the same port. Administrators could however decide to give more priority to web traffic than email: to this end they can use one of the proposed classifiers to mark each of the above connections with a tag that identifies the generating application class. Given the nature of the proposed approaches, administrators have to first train classifiers on pre-recorded traffic traces, each representing one of the $M$ allowed protocols: later, when a new flow comes, the deployed algorithm appends the class tag that is then used by the edge router to speed up the de-queuing of those packets whose class should be prioritized according to a given policy.

We report an example of a QoS policy in Table 1, where protocols have been grouped depending on their usage context. For example, since POP3 and IMAP are both used for message retrieval they are given the same priority. We assign then an higher priority to chat related protocols - one row above, and so on. Note that the last line of the table is not used within this scenario: we will consider it in the next one. To compare the classification performance of the proposed algorithms, we can then introduce a cost matrix: the cost of misclassifying protocol $A$ as protocol $B$ is obtained by simply computing, for each couple $(A, B)$, the distance between their priorities.

\subsection{Known+unknown Classes}

When control on the hosts is released, protocols that have not been considered for training can unexpectedly appear: users can, in fact, upgrade applications or install new ones; mobile users coming from the outside can use the network to connect their 
Table 2. A possible protocol categorization in a Security context

\begin{tabular}{|c|c|c|}
\hline Class & Service & Applications/Protocols \\
\hline accepted traffic & $\begin{array}{c}\text { web } \\
\text { interactive } \\
\text { mail } \\
\text { network management } \\
\text { bulk }\end{array}$ & $\begin{array}{l}\text { http, https, ssh, ftp(control), smtp, pop3, pop3s, } \\
\text { imap, imaps, dns, netbios, smb, snmp, ntp, } \\
\text { ftp(data), sftp (data) }\end{array}$ \\
\hline denied traffic & $\begin{array}{c}\text { chat } \\
\text { p2p } \\
\text { voip } \\
\text { news } \\
\text { streaming multimedia } \\
\text { online gaming } \\
\text { other }\end{array}$ & $\begin{array}{l}\text { IRC, msn messenger, yahoo messenger, AIM, } \\
\text { FastTrack, eDonkey2000, BitTorrent, Gnutella, } \\
\text { OpenNap, Soulseek, Ares, MP2P, Dirrect Con- } \\
\text { nect, GoBoogy, Soribada, PeerEnabler, skype, } \\
\text { WengoPhone, Ekiga, Asterisk, nntp, mms (wmp), } \\
\text { real, quicktime, shoutcast, vbrick streaming, log- } \\
\text { itech Video IM, HalfLife, Counter-Strike, Age of } \\
\text { Empires }\end{array}$ \\
\hline
\end{tabular}

equipment such as laptop or organizers. In both cases a flow generated by a protocol for which no training data was available, could cross the edge router where classification algorithms, as those considered in the previous section, will inevitably assign it to one of the known classes - probably to the class whose behavior is closest to the protocol that is generating this flow.

To avoid to assign not characterized flows to any of the classes chosen for the training, algorithms should be hence partially modified to be given the ability to reject these flows, as explained in Section 2 It is worth noting that erroneous assignments can occur also in this scenario: in this case, however, misclassification not only determines impairments to QoS but can impact also the security of the network. Suppose, in fact, that the administrators of a company network are told to protect some business related information that are commonly accessed by inside users: a first mandatory measure is to prohibit $\mathrm{P} 2 \mathrm{P}$ communications to prevent users to unconsciously export important information through file sharing. Misclassification could hence let a P2P flow pass opening up a hole in the security of the network.

To evaluate the performances of the proposed approaches in these two contexts we consider again error costs, separating the scenario in two sub-cases. In the first we will consider the QoS context: similarly to what described in Section 3.1 we take the same QoS policy as that reported in Table 1. This time the last row expresses the priority associated to flows that do not fall inside any of the classes depicted in the upper rows.

In the second sub-case we will examine the security context. Here we divide protocols in two classes, one composed of protocols that can pass the edge router, the other composed of forbidden protocols. The former class has to be completely formalized and each allowed protocol be described by a distinct subclass. The latter class, instead, is composed of a few protocols, that are explicitly described by separated subclasses, and of all the protocols for which no training data was available. A possible protocol categorization is reported in Table 2 . 


\section{Experimental Results}

\subsection{Dataset Description}

The packet traces used in our experimental evaluation were collected at the border router of University of Brescia's network. For every packet we captured the first 400 bytes of payload and we can apply on these bytes pattern-matching mechanisms to assess the actual application that has generated each TCP flow, in some cases with the addition of manual inspection. The network infrastructure comprises several 1000BaseTX segments routed through a Linux-based dual-processor box and includes about a thousand workstations with different operating systems. All the traffic traces were gathered on the $100 \mathrm{Mb} / \mathrm{s}$ link connecting the edge router to Internet during the first three weeks of June 2007. A total of 50GB of traffic was collected by running Tcpdump for fifteen minutes regularly every hour.

Both the training and the test sets are composed of six different protocol classes. These protocols belong to different application types: web browsing, mail services, $\mathrm{P} 2 \mathrm{P}$ and interactive. The six protocols we select are responsible for the generation of the most part of the traffic, and, given their variety, they allow us to verify the general applicability of our classification techniques. In Table 3 [left] we report the number of flows for the training and the test set. We took care of choosing the training and the test set from traces collected in two separate and consecutive time frames.

In addition to the six protocols mentioned above, we captured another class of flows called other. This class was used during the test phase to verify the classifier's ability to recognize protocols different than those used during the training phase. This subset

Table 3. Left: number of flows included in the considered traces. Right: Cost matrix for the considered protocols. Values in parentheses refer to a Security context, while the other ones to a QoS application.

\begin{tabular}{|c||c|c||c|c|c|c|c|c|c|}
\hline protocol & training set & test set & http & smtp & pop3 & ftp & bitTorrent & msn & unknown \\
\hline \hline http & 7063 & 4928 & - & $2(1)$ & $2(1)$ & $0(1)$ & $5(10)$ & $1(10)$ & $6(10)$ \\
\hline smtp & 19427 & 19480 & $2(1)$ & - & $0(1)$ & $2(1)$ & $3(10)$ & $1(10)$ & $4(10)$ \\
\hline pop3 & 19611 & 19940 & $2(1)$ & $0(1)$ & - & $2(1)$ & $3(10)$ & $1(10)$ & $4(10)$ \\
\hline ftp & 6296 & 14458 & $0(1)$ & $2(1)$ & $2(1)$ & - & $5(10)$ & $1(10)$ & $6(10)$ \\
\hline bitTorrent & 5057 & 7412 & $5(10)$ & $3(10)$ & $3(10)$ & $5(10)$ & - & $4(1)$ & $1(1)$ \\
\hline msn & 1024 & 1033 & $1(10)$ & $1(10)$ & $1(10)$ & $1(10)$ & $4(1)$ & - & $5(1)$ \\
\hline other & & 7912 & $6(10)$ & $4(10)$ & $4(10)$ & $6(10)$ & $1(1)$ & $5(1)$ & - \\
\hline
\end{tabular}

Table 4. Comparison among the three different approaches in different scenarios

\begin{tabular}{c|c|c|c|c|c|}
\cline { 2 - 6 } \multicolumn{1}{c|}{} & \multicolumn{2}{c|}{ Overall Accuracy } & \multicolumn{2}{c|}{ Cost (QoS) } & Cost (Security) \\
\cline { 2 - 6 } & known & known+unknown & known & known+unknown & known+unknown \\
\hline \hline SVM & $93.87 \%$ & $94.72 \%$ & 0.08 & 0.32 & 0.18 \\
\hline GMM & $98.83 \%$ & $97.07 \%$ & 0.02 & 0.13 & 0.20 \\
\hline DT & $98.27 \%$ & $92.53 \%$ & 0.03 & 0.29 & 0.38 \\
\hline
\end{tabular}


is composed by flows that did not match the expressions of the training protocols but matched at least one of the patterns of all the other protocols in [16] (such as edonkey, ssl, smb, gnutella, imap, etc): we are hence relatively sure that they belong to other protocol classes than those listed in Table 3 [left].

By using the considerations made in Section 3 we defined the cost matrices for this dataset (see Table 3 [right]) in both security and QoS contexts. Note that the last row and the last column of this table are not used in the known classes scenario.

\subsection{Analysis of the Classification Results}

Table 4 reports a summary of the results we obtained both for the scenario when only known protocols are tested and in the case known and unknown protocols are classified. In the second case, we fixed to $1.5 \mathrm{e}-14$ and 0.02 the thresholds for the reject option of the GMM and of the DT, respectively.

Generally speaking, we observe that the accuracy of correctly classifying known protocols gets worse when unknown classes are introduced. This surely happens with the GMM and the DT approaches since some of the flows that are correctly reported as their actual classes in the first scenario, can be mapped to the unknown class in the second: e.g., all flows that are correctly classified by a GMM when there are no unknown classes and that get a value of the a posteriori probability lower than the threshold, are misclassified. While this behavior is intrinsic for these two approaches, the recognition rate for the various protocols does not necessarily decrease when the SVM architecture is used. As explained, in fact, in Section 2.1 the SVM architecture completely changes when unknown classes are introduced.

We point out now some differences between the three approaches, considering the scenario with unknown protocols. In particular, the design of the SVM-based classifier presents characteristics differing from the other two architectures: $i$ ) it is the only classifier that does not require to be trained on samples coming from unknown classes; $i i$ ) while thresholds in the GMM and DT classifiers have the same value for all the training protocols, we find the one-class SVM parameters independently for each protocol and iii) finally the models of all application classes for both the GMM and DT approaches have been determined on the same $n$-dimensional space (a fourth dimensional space has been chosen in both approaches), while SVM builds each one-class model on the space that allows better classification results for that class (the value of $n$ varied between two and six). This optimization process for the SVM approach leads to better results in classifying unknown flows when compared to the other two techniques even if the accuracy of all the proposed techniques are very similar as reported in the second column of Table 4

Finally, a note on accuracy, which cannot be used as a single quality parameter for a classifier. Even if the GMM always exhibited the best accuracy (Table 4), in fact, there is a scenario (Security with unknown classes) in which the cost achieved by an SVM is lower. Furthermore, if we are interested in an on-line implementation, DT could be also a good choice since it performs acceptably, especially in a QoS scenario. 


\section{Conclusions}

In this paper we presented three approaches to the classification of IP flows, based on Support Vector Machines, Gaussian Mixture Models and Decision Trees. One of the problems that arise in this context is that the classifiers need to deal with the sudden appearance of new application protocols. We proposed to address this problem by rejecting a pattern that does not fit to any of the known classes, by means of a suitably defined distance-based reject option. A further contribution of this work has been the definition of different network scenarios and the proposal of a cost matrix for each of them so as to weight in a different way the various classification errors. This permitted a more effective comparison of the proposed approaches on real traffic traces.

In the future we plan to implement the proposed classification architectures in an on-line system allowing the comparison of their computational requirements as well as their behavior when applied to different network scenarios.

\section{References}

1. Skype, http://www.skype.com

2. Karagiannis, T., Papagiannaki, K., Faloutsos, M.: BLINC: multilevel traffic classification in the dark. In: Proceedings of the 2005 conference on Applications, technologies, architectures, and protocols for computer communications (SIGCOMM 2005), Philadelphia, PA, USA, pp. 229-240 (August 2005)

3. Roughan, M., Sen, S., Spatscheck, O., Duffield, N.: Class-of-service mapping for QoS: a statistical signature-based approach to IP traffic classification. In: Proceedings of the 4th ACM SIGCOMM conference on Internet measurement (IMC 2004), Taormina, Sicily, Italy, pp. 135-148 (October 2004)

4. McGregor, A., Hall, M., Lorier, P., Brunskill, J.: Flow Clustering Using Machine Learning Techniques. In: Barakat, C., Pratt, I. (eds.) PAM 2004. LNCS, vol. 3015, pp. 205-214. Springer, Heidelberg (2004)

5. Williams, N., Zander, S., Armitage, G.: A Preliminary Performance Comparison of Five Machine Learning Algorithms for Practical IP Traffic Flow Classification. SIGCOMM Computer Communication Review 36(5), 7-15 (2006)

6. Bernaille, L., Teixeira, R., Salamatian, K.: Early Application Identification. In: Proceedings of the 2006 ACM CoNEXT conference (CoNEXT 2006), Lisboa, Portugal, pp. 1-12 (December 2006)

7. Li, R.Y.Z., Guan, X.: Accurate Classification of the Internet Traffic Based on the SVM Method. In: Proceedings of the 42th IEEE International Conference on Communications (ICC 2007), Glasgow, Scotland, pp. 1373-1378 (June 2007)

8. Tax, D.M.J., Duin, R.P.W.: Growing a multi-class classifier with a reject option. Pattern Recognition Letters 29, 1565-1570 (2008)

9. Fumera, G., Roli, F., Giacinto, G.: Reject option with multiple thresholds. Pattern Recognition 33(12), 2099-2101 (2000)

10. De Stefano, C., Sansone, C., Vento, M.: To reject or not to reject: That is the question - an answer in case of neural classifiers. IEEE Trans. on Systems, Man and Cybernetics 30(1), 84-94 (2000)

11. Landgrebe, T., Tax, D.M.J., Paclík, P., Duin, R.P.W.: The interaction between classification and reject performance for distance-based reject-option classifiers. Pattern Recognition Letters 27(8), 908-917 (2006) 
12. Vapnik, V.N.: Statistical Learning Theory. John Wiley and Sons, New York (1998)

13. Schölkopf, B., Platt, J.C., Shawe-Taylor, J., Smola, A.J., Williamson, R.C.: Estimating the Support of a High-Dimensional Distribution. Neural Computation 13, 1443-1471 (2001)

14. Duda, R.O., Hart, P.E., Stork, D.G.: Pattern Classification, 2nd edn. Wiley, Chichester (2000)

15. Rissanen, J.: Modeling by shortest data description. Automatica 14, 465-471 (1978)

16. L7 Filter, http://17--filter.sourceforge.net 